\title{
Fluctuating magnetic moments in liquid metals
}

\author{
Mark Patty, Keary Schoen, and Wouter Montfrooij \\ Department of Physics and Astronomy, and Missouri Research Reactor, University of Missouri, Columbia, Missouri 65211, USA
}

(Received 23 June 2005; published 27 February 2006)

\begin{abstract}
We reanalyze literature data on neutron scattering by liquid metals and show that there is an additional broad (in energy) quasielastic mode present that is absent in x-ray scattering. This mode cannot be accounted for by the standard coherent and incoherent scattering mechanisms. We argue that this mode indicates that nonmagnetic liquid metals possess a magnetic moment which fluctuates on a picosecond time scale. This time scale is the same as the time scale of the cage-diffusion process in which an ion rattles around in the cage formed by its neighbors. We find that these fluctuating magnetic moments are present in liquid $\mathrm{Hg}, \mathrm{Al}, \mathrm{Ga}$, and $\mathrm{Pb}$ and possibly also in the alkali metals.
\end{abstract}

DOI: 10.1103/PhysRevE.73.021202

PACS number(s): 66.10.-x, 61.25.Mv, 72.15.Cz

\section{INTRODUCTION}

During the past decades the properties of a range of elemental liquid metals have been studied by means of neutron scattering [1-27] and x-ray scattering experiments [28-38]. Unlike ordinary simple fluids, liquid metals can support short-wavelength sound waves far outside the hydrodynamic regime; simple fluids only support very strongly damped density fluctuations beyond the hydrodynamic region [39]. In other words, a density disturbance decays much faster in a simple fluid than it does in a liquid metal under comparable thermodynamic conditions. Typically, a short-wavelength sound wave in a simple liquid does not propagate beyond one wavelength [39]. Presumably, this difference can be attributed to the presence of two interacting systems in a liquid metal: the positively charged ionic liquid and the negatively charged conduction sea. This notion has stimulated the study of the decay mechanism of the density fluctuations by means of neutron and x-ray scattering experiments, as well as by molecular dynamics (MD) computer simulations in a range of liquid metals, such as $\mathrm{Hg}[1-3,28,29]$, Cs $[4,5,30]$, $\mathrm{K}$ [6-8], $\mathrm{Rb}[9,10], \mathrm{Na}[11-14,31,32], \mathrm{Li}[15-17,33], \mathrm{Pb}$ $[18-21,34]$, Al $[22,35,36]$, and Ga [23-25,37,38]. These studies have, by and large, confirmed the role of the electron sea as a feedback mechanism, serving to reduce the decay rates of disturbances and ensuring that density fluctuations can propagate at a higher velocity than the adiabatic sound velocity.

These studies also showed that, similar to ordinary liquids, cage diffusion plays an important part in the decay mechanism of density fluctuations [3,39-43]. Cage diffusion occurs when an atom bounces off neighboring atoms, thereby confining the atom to a "cage." This is in contrast to self-diffusion, the process in which the atom moves through the sample and which is characterized by a net displacement from its starting position over a period of time [39]. In MD simulations, where one follows the position of an atom over time, cage diffusion and self-diffusion show up as two distinct time scales [1]. Cage diffusion accounts for a small decrease in correlation between the initial and subsequent positions of an atom; this initial decrease in correlation occurs within a few picoseconds. The overall demise of correlation is given by the self-diffusion process, which takes place on a much longer time scale [39] and is determined by the coefficient for self-diffusion, $D_{s}$. These two diffusive processes can also be observed by means of quasielastic neutron scattering [2]. Neutron scattering is sensitive to the motion of individual atoms because an atom moves during the time it takes the neutron to interact with it [44]. This motion shows itself as a spread in energy of the scattered neutron wave packet. Rapid movement (cage diffusion) results in a large spread in energy; slow movement (self-diffusion) results in a spread with small characteristic energy width. Both processes have indeed been observed in liquid metals. For instance, in liquid mercury [1-3], the scattered neutron intensity originating from a single atom (the so-called incoherent scattering contribution [44]) corresponds to a superposition of two Lorentzian lines. One line is sharp (in energy), corresponding to self-diffusion, and one line is broad, corresponding to cage diffusion. A Lorentzian line in energy corresponds to an exponential decay in time [44] of the correlation between the initial and subsequent positions of an atom.

A comparison between the neutron scattering data and the MD simulations on liquid $\mathrm{Hg}$ revealed a serious discrepancy regarding the effectiveness of the cage-diffusion mechanism [1-3]. While both studies agreed on the characteristic time scale for the cage-diffusion process, according to the neutron scattering study [2] cage diffusion accounted for up to $20 \%$ of the loss in correlation in the position of an atom, compared to only $0.4 \%$ as observed in the MD results [3]. In order to explain this discrepancy, Badyal et al. [2] suggested that a mercury ion might have a fluctuating magnetic moment, resulting in an enhanced neutron scattering cross section. The idea here is straightforward (see Fig. 1): in a liquid, atoms can approach each other very closely. On such a close approach, an electron from a filled inner shell of the metallic ion can be ejected into the Fermi sea [Fig. 1(b)], resulting in an unpaired electron, and hence in a magnetic moment [Fig. $1(\mathrm{c})]$. Once the ions move away from each other again, the shell can be recompleted [Fig. 1(d)]. One can thus expect a magnetic moment to pop in and out of existence on the same time scale as the rattling motion of an atom inside its cage. This process automatically leads to a pathway for the neutron to scatter from the atom via the electromagnetic force [44], augmenting the interaction via the strong nuclear force and 


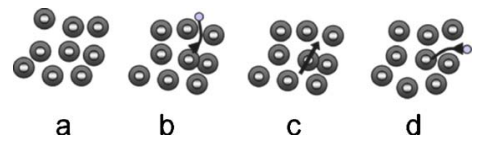

FIG. 1. (Color online) Schematic representation of how cage diffusion can lead to short-lived magnetic moments. (a) Snapshot of a metallic liquid with ions showing completely filled shells. The Fermi sea is not shown. (b) On close approach an electron is kicked out of an orbital. (c) The resulting unpaired electron leads to a local magnetic moment. (d) This moment disappears again as the atoms move away from each other.

resulting in an enhanced cross section for the cage-diffusion process. From the strength of the magnetic interaction [44], it can then be determined what fraction of the time an ion has an unpaired electron.

In this paper we show that the cage-diffusion process in liquid metals is indeed accompanied by a fluctuating magnetic moment. We do this by reexaming published neutron scattering data on $\mathrm{Hg}$, Cs, K, Rb, Na, Li, Pb, Al, and Ga. We observe a small effect in the alkali metals, but find that the ions in $\mathrm{Ga}$ and $\mathrm{Hg}$ have unpaired electrons for up to $20 \%$ of the time. Not only do these magnetic moments provide an additional means for studying cage diffusion by means of neutron scattering, they provide an additional long-range interaction mechanism for the ions in the liquid.

\section{THEORY}

In this section we briefly review the various contributions that make up the neutron scattering cross section of a liquid. We use the data by Badyal et al. [2] on mercury to illustrate the various contributions and to demonstrate under what conditions one can observe the proposed fluctuating magnetic moments.

A neutron interacts with the nucleus of an atom via the strong nuclear force and with the magnetic moments of electrons present in the system via the electromagnetic force [44]. Thus, the total number of neutrons with initial energy $E_{i}$ that are scattered every second into a solid angle $d \Omega$ having final energies between $E_{f}$ and $E_{f}+d E$ is given by the double-differential cross section and can be separated into a nuclear and a magnetic term [44]:

$$
\frac{d^{2} \sigma_{\text {total }}}{d \Omega d E}=\frac{d^{2} \sigma_{\text {nuclear }}}{d \Omega d E}+\frac{d^{2} \sigma_{\text {magnetic }}}{d \Omega d E} .
$$

For monoatomic systems, such as the ones considered in this paper, the nuclear contribution for single scattering events is given by

$$
\frac{d^{2} \sigma_{\text {nuclear }}}{d \Omega d E}=\frac{k_{f}}{k_{i}} \frac{\sigma_{c o h}}{4 \pi} S_{c o h}(q, E)+\frac{k_{f}}{k_{i}} \frac{\sigma_{i n c}}{4 \pi} S_{\text {inc }}(q, E) .
$$

$S_{c o h}(q, E)$ is the dynamic structure factor and represents the collective response of the liquid as a function of momentum $\hbar q$ and energy $E$ transferred from the neutron to the liquid, while $S_{\text {inc }}(q, E)$ describes the dynamics of a single atom [44]. The cross sections $\sigma_{c o h}$ and $\sigma_{i n c}$ are element dependent; $\sigma_{i n c}$ arises because the strong interaction depends on the spin

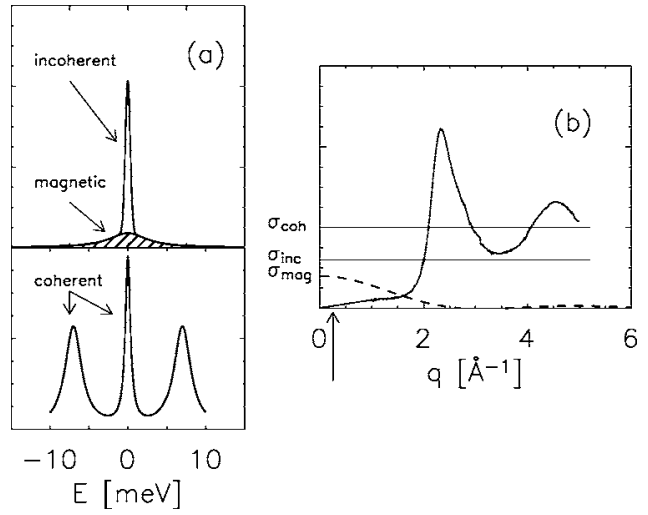

FIG. 2. (a) A schematic representation of the three contributions that make up the scattering by a liquid at low $q$ : the incoherent, magnetic, and coherent contributions. The incoherent contribution consists of a sharp central line representing self-diffusion, plus a broad central cage-diffusion mode whose intensity is so low (less than $0.5 \%$ ) that it is not visible in the figure. The magnetic scattering shows up as a broad central line, roughly independent of $q$. Its intensity is element dependent, but is typically of the same order as the coherent contribution. The coherent contributions consists of the Rayleigh-Brillouin triplet at low $q$. Similar to the incoherent contribution, these three peaks sit on top of a broad cage-diffusion mode whose intensity is too small to show up in this figure. (b) The relative strength of the three contributions depends on the momentum transfer, as well as on element dependent cross sections. The arrow indicates the low- $q$ value used in part (a) where the magnetic contribution is most prominent [47].

state of the nucleus and the number of neutrons in the nucleus. Thus, the nuclear scattering cross section carries information about the collective behavior of the atoms, such as sound waves, and information about the motion of indiviual atoms, such as self-diffusion. [44]

The static structure factor $S(q)$ is given by the sum rule

$$
S(q)=\int S_{c o h}(q, E) d E,
$$

while the incoherent dynamic structure factor satisfies a similar sum rule

$$
1=\int S_{i n c}(q, E) d E .
$$

The various contributions are illustrated in Fig. 2. In neutron diffraction experiments aimed at measuring $S(q)$, the energy integration in Eq. (3) is carried out by the neutron detector. Because of the term $k_{f} / k_{i}$ in Eq. (2), this procedure leads to small errors in the determination of $S(q)$; however, these errors are small under suitable experimental conditions and can be corrected for using standard methods [44]. A further source of errors is that Eq. (2) is only valid for neutrons that are scattered once by the sample. Again, by choosing sufficiently small samples, the errors introduced by multiplescattering events and events in which a scattered neutron is absorbed by the sample can be corrected for [45]. Therefore, provided these corrections have been carried out, one can 
check the accuracy of the data reduction procedure by comparing the measured cross sections $\sigma_{c o h}$ and $\sigma_{i n c}$ to the known values. Even in the case where the absolute values of $\sigma_{c o h}$ and $\sigma_{i n c}$ cannot be inferred from the experiment, their ratio can still be determined using the fact that $S(q)$ oscillates around 1 for large $q$.

The magnetic contribution to the scattered intensity is only visible in neutron scattering experiments on liquids provided that atoms with unpaired electrons exist [44]. The angular momentum associated with these unpaired electrons, $h J$, interacts with the intrinsic magnetic moment of the neutron. The conduction electrons present in liquid metals do not contribute to the scattering at finite $q$; an electron moves so fast compared to the neutron that the scattered waves only add up coherently at $q=0$, the forward direction. However, if an electron is localized around an atom, all scattered waves originate from the region of the partially filled orbital and the scattered waves can be observed for a range of $q$ values. For this reason the form factor for magnetic scattering $F(q)$, which describes the variation of scattered intensity with $q$ and which is given by the spatial extent of the electron cloud, falls off more rapidly with increasing $q$ than the form factor for nuclear scattering [the so-called Debye-Waller factor $W(q)]$. The latter reflects the fact that nuclear scattering originates in the much smaller volume of the nucleus.

The number of ions with unpaired electrons at any given moment determines the magnetic cross section for a liquid with fluctuating magnetic moments. The total number of neutrons that are scattered per second per metallic ion into solid angle $d \Omega$ is given by the paramagnetic approximation for the differential cross section [44]:

$$
\frac{d \sigma^{\text {magnetic }}}{d \Omega}=n \frac{2}{3}\left(\gamma r_{0}\right)^{2}\left[\frac{1}{2} g(L J S) F(q)\right]^{2} e^{-2 W(q)} J(J+1) .
$$

In this equation, $n$ is the fraction of the ions that have a collision-induced angular momentum $h J, \quad g(L J S)=3 / 2$ $+[S(S+1)-L(L+1)] /[2 J(J+1)]$ describes how the intrinsic angular momentum of the electron, $h S$, and its orbital angular momentum $h L$ add up to the magnetic moment $\mu_{B} g(L J S) J$ ( $\mu_{B}$ is one Bohr magneton), and $\left(\gamma r_{0}\right)^{2}=0.291 \mathrm{~b}$ is the strength of the interaction with the neutron. Equation (5) offers a good approximation of the strength of the magnetic scattering provided that the characteristic energy width of the quasielastic scattering as determined by the underlying cage-diffusion mechanism is small compared to the incident energy of the neutron [44]. This is the same requirement that allows one to determine $S(q)$ from a liquid without doing an energy analysis of the scattered neutron, and we will therefore assume that this requirement is satisfied for all published data sets discussed in this paper.

Analyzing quasielastic neutron scattering experiments on liquid $\mathrm{Hg}$ at room temperature, Badyal et al. [2] observed that the scattered signal at small momentum transfers consisted of two contributions (see Figs. 2 and 3), attributable to self-diffusion and cage diffusion, respectively. However, the relative strength (area under the curves in Fig. 3) of the cagediffusion contribution compared to the self-diffusion contri-

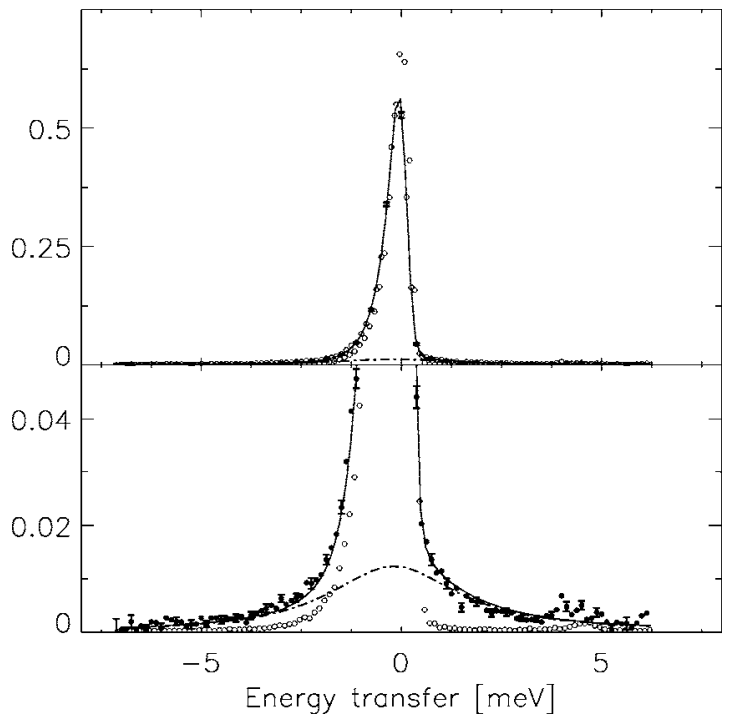

FIG. 3. The dynamic structure factor of liquid mercury [2] at small momentum transfer (solid circles) and a vanadium reference sample (open circles) showing the resolution of the neutron scattering spectrometer. The solid line is a fit to two Lorentzian lines, taking the asymmetric spectrometer resolution function into account. The bottom figure is an enhancement of the top figure. One observes a sharp (in energy, hence slow in time) central mode reflecting self-diffusion and a broad mode (dash-dotted curve) reflecting the fast rattling motion of an atom inside the cage formed by its neighbors. The intensity of this broad mode (clearly absent in the vanadium spectra) was found to be larger [2] by a factor of 20 than could be expected from nuclear sum rules on the scattering. Hence, the intensity was attributed to a paramagnetic cross section, reflecting an unpaired $d$ electron on a time scale determined by cage diffusion (figure reproduced from Ref. [2]).

bution was found to be $22 \%$ (corresponding to a differential cross section of $\sim 1.5 / 4 \pi$ barn). A relative strength of the order of $0.3 \%$ was expected based on MD simulations [3] and on an order-of-magnitude calculation [2]. Given that the strength of the quasielastic coherent contribution for small $q$ values (given by sum rules at $\sim 0.01 / 4 \pi$ barn) was negligible $[44,46]$ and given that the characteristic energy width $\left(3 \mathrm{ps}^{-1}\right)$ corresponded to the time scale of the cage-diffusion process $(1 / 3 \mathrm{ps})$, the authors [2] concluded that the broad quasielastic line did indeed correspond to cage diffusion but with a magnetically enhanced cross section (depicted in Fig. 2). Using Eq. (5) $[S=1 / 2, L=2$, and $J=5 / 2$ and $F(q)=e^{-W(q)}=1$ for small $\left.q\right]$ and noting that crystal electric field effects are absent in a liquid, we find that $20 \%$ of the $\mathrm{Hg}$ ions have an unpaired $d$ electron. Should the observed magnetic signal originate from an unpaired $s$ electron, then the corresponding fraction of magnetic ions would be $82 \%$. We return to this latter possibility in the Discussion.

Thus, a significant fraction of the mercury ions has a magnetic moment; this moment can interact with its neighbors via the magnetic-dipole interaction via the direct exchange interaction and via polarization of the conduction electrons. The dipole interaction likely only adds up to a small correction to the interatomic potential at small distances, but it becomes the dominant interaction mechanism at large dis- 
TABLE I. The observed magnetic cross section $\sigma_{m a g n}$ and the corresponding fraction $n$ of ions with a magnetic moment, calculated for the most likely quantum numbers of the unpaired electron using Eq. (5).

\begin{tabular}{|c|c|c|c|c|c|c|c|c|}
\hline Element & $T / T_{\text {melting }}$ & $S$ & $L$ & $J$ & $g(L J S)$ & $\begin{array}{c}\sigma_{\text {magn }} \\
{[\mathrm{b}]}\end{array}$ & $\begin{array}{c}n \\
{[\%]}\end{array}$ & Ref. \\
\hline $\mathrm{Li}$ & 1.03 & $1 / 2$ & 0 & $1 / 2$ & 2 & $0-0.01$ & $0-1$ & [15] \\
\hline \multirow[t]{2}{*}{$\mathrm{Al}$} & 1.003 & $1 / 2$ & 0 & $1 / 2$ & 2 & 0.16 & 9 & [22] \\
\hline & & $1 / 2$ & 1 & $3 / 2$ & 1.33 & & 4 & \\
\hline \multirow[t]{8}{*}{$\mathrm{Ga}$} & 1.08 & $1 / 2$ & 0 & $1 / 2$ & 2 & 0.4 & 22 & [24] \\
\hline & & $1 / 2$ & 2 & $5 / 2$ & 1.2 & & 5.2 & \\
\hline & 1.08 & $1 / 2$ & 0 & $1 / 2$ & 2 & 0.88 & 48 & [23] \\
\hline & & $1 / 2$ & 2 & $5 / 2$ & 1.2 & & 12 & \\
\hline & 3.17 & $1 / 2$ & 0 & $1 / 2$ & 2 & 0.78 & 42 & [23] \\
\hline & & $1 / 2$ & 2 & $5 / 2$ & 1.2 & & 10 & \\
\hline & 3.20 & $1 / 2$ & 0 & $1 / 2$ & 2 & 0.5 & 27 & [24] \\
\hline & & $1 / 2$ & 2 & $5 / 2$ & 1.2 & & 6.5 & \\
\hline $\mathrm{Cs}$ & 1.02 & $1 / 2$ & 1 & $3 / 2$ & 1.33 & 0.11 & 2.7 & {$[4]$} \\
\hline \multirow[t]{2}{*}{$\mathrm{Hg}$} & 1.25 & $1 / 2$ & 0 & $1 / 2$ & 2 & 1.5 & 82 & {$[2]$} \\
\hline & & $1 / 2$ & 2 & $5 / 2$ & 1.2 & & 20 & \\
\hline \multirow[t]{2}{*}{$\mathrm{Pb}$} & 1.02 & $1 / 2$ & 0 & $1 / 2$ & 2 & 0.7 & 38 & [21] \\
\hline & & $1 / 2$ & 2 & $5 / 2$ & 1.2 & & 9 & \\
\hline
\end{tabular}

tances; therefore, it might well contribute to the ability of a liquid metal to sustain propagating sound waves with short wavelengths. Likewise, polarization of the conduction electrons by the atomic magnetic moments provides a direct interaction mechanism between the ionic liquid and the conduction electrons. It is the presence of the two interacting systems that is presumably responsible for the existence of well-defined short-wavelength sound waves. For this reason, we have reanalyzed existing neutron scattering data $[4,6,15,21-24]$ on liquid metals in order to investigate the presence of magnetic moments in nonmagnetic liquids. We note that short-lived magnetic moments do not contradict the overall diamagnetic response of a liquid metal: macroscopic measurements take place on a much larger time scale than the lifetime of a collision-induced atomic moment.

Fluctuating magnetic moments can betray their presence in various ways in neutron scattering experiments. In diffraction experiments the additional cross section would lead to an increased signal at smaller $q$ values, decaying with $q$ according to $|F(q)|^{2}$. This additional signal would be on top of the angle-independent incoherent cross section and the weakly angle-dependent multiple-scattering cross section. Thus, whether the proposed signal is actually visible in the published data depends on the strength of the incoherent cross section and on the details of the data reduction procedure. It is easiest to identify the magnetic cross section in quasielastic neutron scattering experiments (as in liquid mercury experiments [2]); however, we found only one data set [24] in the literature suited to the latter approach. Finally, it is unclear a priori how an increase in temperature and density would affect the magnetic cross section. This increase would allow for a closer approach of the ions, thereby increasing the overlap of the filled orbitals; however, the lifetimes of the induced moments would likely decrease as well, resulting in a signal that would be too spread out in energy to be reliably observable in neutron scattering experiments.

\section{RESULTS}

Our investigation is limited to published studies that show the raw data and detail the correction procedure or to studies where the incoherent scattering contribution is absent. Surprisingly, this leaves very few data sets on liquid metals. In most investigations the data are only presented after subtraction of the contribution identified as incoherent scattering. This subtraction procedure would also have eliminated the magnetic contribution, should it have been present. Evaluation of the published neutron scattering data on the much studied alkali metals shows that the percentage of ions having a magnetic moment is likely to be much smaller than what was observed in liquid mercury. In most cases it is not possible to come to an unambiguous conclusion whether this magnetic contribution is present or not. On the other hand, the group- 3 and -4 metals $\mathrm{Al}, \mathrm{Pb}$, and $\mathrm{Ga}$ show a large effect similar to liquid mercury. All results are collected in Table I.

Before discussing the neutron scattering results in detail, we note that inelastic x-ray scattering experiments [28-38] on a variety of liquid metals do not show evidence for an additional mode. Given the accuracy of recent experiments and the fact that only the coherent contribution is measured in X-ray scattering experiments, this rules out the possibility that any additional mode observed in neutron scattering experiments is part of the coherent scattering (Fig. 2). This is as expected from MD simulations [3,25]: while the decay in correlation due to cage diffusion is visible in the coherent dynamics as a decline in correlation that happens over a short period of time, its intensity (magnitude of the decline) is so small $(<0.5 \%$ at small $q$ values [47]) that it cannot be 
observed as a separate mode in the coherent contribution. Thus, the effects of cage diffusion can be observed in the linewidth of the scattering spectra [36,37], but cage diffusion does not show up as a separate contribution (mode) to the scattering.

\section{A. Alkali metals}

Bodensteiner et al. [4] observed a discrepancy between the value for the incoherent scattering cross section as measured in their inelastic neutron scattering experiments on liquid cesium at $308 \mathrm{~K}$ and the commonly accepted value. After having accounted for all corrections to the normalization of the neutron scattering data, Bodensteiner et al. [4] inferred a (total) incoherent cross section of $0.33 \mathrm{~b}$ instead of the literature value of $0.22 \mathrm{~b}$. Assuming that $0.22 \mathrm{~b}$ is indeed the correct value for the incoherent cross section, this would imply a magnetic cross section of $0.11 \mathrm{~b}$, or $d \sigma^{\text {magnetic }} / d \Omega$ $=0.11 / 4 \pi$. Presumably, a collision would leave a cesium ion temporarily with an iodine configuration $[S=1 / 2, L=1$, $J=3 / 2$, and $g(L J S)=4 / 3$ ], yielding $n=2.7 \%$ [see Eq. (5)]. Unfortunately, since uncorrected spectra at the smallest $q$ values $\left(q<0.5 \AA^{-1}\right)$ were not published in this study [4], we could not infer whether the supposed magnetic cross section indeed corresponded to a quasielastic spectrum characterized by a cage-diffusion linewidth.

From the current literature results, it is inconclusive whether liquid potassium [6-8], liquid rubidium [9,10], or liquid sodium [11-14,31,32] displays a magnetic cross section. Either the data at low $q$ are not accurate enough or not enough details of the data correction procedure have been given to test our hypothesis. Bearing in mind the results for liquid cesium, the magnetic cross section of $0.1 \mathrm{~b}$ might just be too small to be observable in sodium $\left(\sigma_{i n c}=1.67 \mathrm{~b}\right)$ and rubidium $\left(\sigma_{\text {inc }}=0.48 \mathrm{~b}\right)$. However, the paramagnetic cross section might have been observed in liquid potassium $\left(\sigma_{i n c}\right.$ $=0.27 \mathrm{~b}$ ) in a series of quasielastic neutron scattering experiments [6]. Cabrillo et al. [6] combined a high- (energy) resolution study on liquid $\mathrm{K}$ at $343 \mathrm{~K}$ with a lower-resolution experiment to model the full dynamic response of potassium down to small $q \sim 0.4 \AA^{-1}$. Doing so, they were able to show that the quasielastic component at small $q$ consisted of two contributions, one corresponding to self-diffusion and one to a process with a lifetime $\tau \sim 3$ ps. Qualitatively, this is similar to the observations for cage diffusion in liquid mercury. Unfortunately, the authors did not give the ratio between the narrow and broad components, making it impossible to infer $n$ from their data. In fact, the authors did not attribute this broad mode to cage diffusion. Instead, it was assumed to be part of the coherent scattering contribution. The latter is inconsistent with their modeling of the rest of the scattered intensity [6], which already completely exhausted the coherent sum rule [Eq. (3)]. Given this and given the very weak dependence of $\tau$ on $q$ for $q<1.3 \AA^{-1}$, we believe that this broad mode represents cage diffusion. However, whether it is a cage-diffusion process combined with a fluctuating magnetic moment cannot be inferred from this study (as published).

Neutron scattering results for liquid lithium leave open the possibility of a magnetic cross section being present, al-

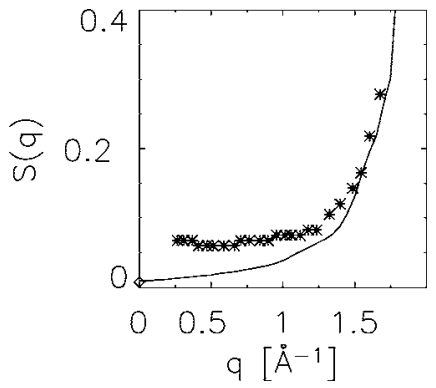

FIG. 4. The static structure factor of liquid lead as measured by x-ray scattering data [34] at $623 \mathrm{~K}$ (solid line) and neutron scattering data [21] at $613 \mathrm{~K}$ (stars). Note the difference between the two data sets at small momentum transfer; the x-ray scattering data approach $S(q=0)=0.008$ (open diamond), while the neutron scattering data approach a constant value well in excess of $S(q=0)$, indicative of a magnetic contribution to the scattering.

beit that the results are somewhat inaccurate owing to the large absorbtion cross section. For instance, Torcini et al. [15] report $S(q=0)=0.04$ at $450 \mathrm{~K}$, while the expected $S(q=0)$ from the compressibility sum rule is 0.03 , thus indicating the presence of a small magnetic cross section. However, not all studies are in agreement with these neutron scattering data (probably due to the large absorbtion cross section for neutrons). Therefore, we can only give an estimated range for the fraction $n$ of ions with an unpaired electron. Based on the work of Torcini et al. [15], we find the fraction $n$ to be in the range $0<n<1 \%$ for $S=1 / 2, L=0$, $J=1 / 2$, and $g(L J S)=2$.

In all, the alkali metals do not show unambiguous evidence for the existence of the proposed magnetic cross section. However, it is interesting to note that small-angle $\mathrm{x}$-ray scattering experiments on liquid lithium indicated the presence of an additional cross section [16], which the authors tentatively attributed to increased correlation between the valence electrons. The mechanism proposed in this paper would offer an explanation for the observed [16] increased correlation. Nonetheless, the evidence for a collision-induced fluctuating moment in the alkali metals is somewhat weak. Much better evidence for its existence comes from scattering experiments on group- 3 and -4 metals, which display an enhanced cross section, similar to the results for liquid mercury.

\section{B. Group-3 and -4 metals}

Liquid lead is a good candidate to analyze for the possible presence of a magnetic cross section since $\mathrm{Pb}$ has a negligible incoherent cross section; therefore, any significant scattering at small momentum transfers (where the coherent cross section is very small) is indicative of a paramagnetic signal. Reijers et al. [21] measured the static structure factor of liquid lead at $613 \mathrm{~K}$ under ambient pressure (see Fig. 4). From Eqs. (2) and (3), we find that the expected neutron scattering intensity at small momentum transfers due to coherent scattering is given by $\sigma_{c o h} /(4 \pi) S(q=0)$, with $S(q=0)=0.009$ [48] and $\sigma_{c o h}=11.16 \mathrm{~b}$. The $S(q=0)$ extrapolated value from the liquid lead experiment is 0.07 (see Fig. 


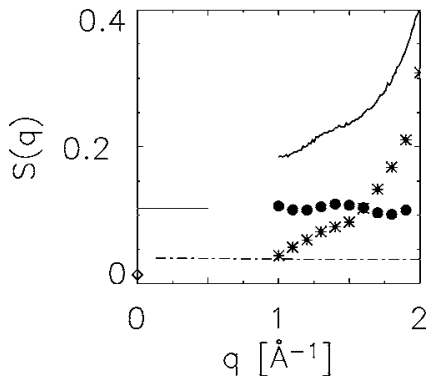

FIG. 5. The static structure factor of liquid aluminum just above the melting point as measured by neutron scattering [22] (solid line) and x-ray scattering [35] (stars). The difference between the two data sets is considerably larger than the calculated multiplescattering contribution to the neutron scattering data (dash-dotted curve). After correcting for these multiple-scattering effects, we find that the remaining difference between the two data sets (solid circles and horizontal line) is only weakly dependent on $q$, indicative of an incompletely filled electronic shell with small radius. The data point at $q=0$ (open diamond) is the compressibility limit taken from thermodynamic data [50].

4), implying an additional neutron scattering intensity of $0.7 / 4 \pi$ barn. Using Eq. (5) with $S=1 / 2, L=2, J=5 / 2$, and $g(L J S)=1.2$, the fraction $n$ of ions with an unpaired electron is $9 \%$. Assuming the additional cross section originates from $s$ electrons $[S=1 / 2, L=0, J=1 / 2$, and $g(L J S)=2]$, we find $n=38 \%$ (See Table I).

Liquid aluminum also displays a paramagnetic cross section. Iqbal et al. [22] performed a study of liquid aluminum at $936 \mathrm{~K}$ (see Fig. 5). In this study on a liquid with negligible incoherent cross section, the authors normalized their data to $S(q \rightarrow \infty)=1$; however, the data had not been corrected for multiple-scattering effects, which can constitute a major part of the scattering at small $q$. Based on the dimensions of their cylindrical cell, we have calculated $[2,45]$ the multiplescattering contribution (dashed line in Fig. 5) assuming the energy dependence of $S(q, E)$ to be given by a Lorenzian line shape with half width determined by the coefficient for selfdiffusion $\left(D_{s}=0.4 \AA^{2} / \mathrm{ps}\right.$ [49]). After subtracting the multiple-scattering contribution and renormalizing the data accordingly, we find that the neutron scattering data consistently lie above the x-ray data [35] at small $q$ and that the neutron scattering data do not appear to reach the $q \rightarrow 0$ limit $S(q=0)=0.013$ [50]. Since a paramagnetic contribution represents a very small correction to $\mathrm{x}$-ray scattering data, we take the difference $\Delta S=0.11$ between the neutron and X-ray $S(q)$ measurements at $q<1.5 \AA^{-1}$ as the strength of the paramagnetic signal-i.e., $d \sigma^{\text {magnetic }} / d \Omega=\Delta S \sigma_{\text {coh }} / 4 \pi$ $=0.16 / 4 \pi$ barn. This corresponds $[\mathrm{Eq}$. (5)] to a fraction $n=4 \%$ assuming the fluorine electronic configuration for paramagnetic Al ions; a sodium configuration would correspond to $n=9 \%$ (see Table I). Again, very accurate inelastic $\mathrm{x}$-ray scattering experiments on liquid $\mathrm{Al}$ [36] did not find evidence for an additional mode (a fourth contribution in addition to the three modes shown in Fig. 2), ruling out the possibility that the additional scattering is part of the coherent contribution.

Another liquid metal for which we can verify the presence of an additional component to the cross section is liquid

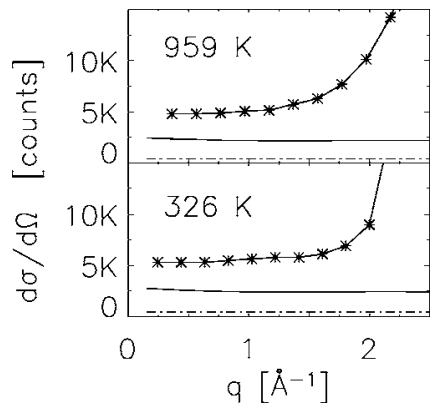

FIG. 6. The unnormalized static structure factor of liquid gallium at two temperatures (solid line with stars) as measured by neutron scattering [23]. The calculated incoherent contribution is given by the dash-dotted lines; the sum of the incoherent and multiple-scattering contribution (see text) is denoted by the solid lines. The difference (at small $q$ values) between the experimental data points and the solid line is ascribed to paramagnetic scattering.

gallium. In fact, gallium is probably the most compelling case of the ones presented in this paper. Bellissent-Funel et al. [23] found in their experiments on liquid $\mathrm{Ga}$ at $326 \mathrm{~K}$ and $959 \mathrm{~K}$ that the observed scattered intensities were not consistent with the known values for $\sigma_{i n c}$ and $\sigma_{c o h}$. Since both uncorrected and corrected data were published in this study [23], and since every step of the data reduction procedure was clearly described, we can infer a very accurate estimate of the paramagnetic cross section for Ga. Using the dimensions of the sample cell used in the experiments [23], we have calculated $[2,45]$ the multiple-scattering contribution (see Fig. 6). Taking into account the $S(q=0)$ values and the fact that the magnetic contribution will be absent at very large $q$, we find an additional differential scattering cross section of $0.88 / 4 \pi$ barn at $326 \mathrm{~K}$ and $0.78 / 4 \pi$ barn at $959 \mathrm{~K}$. Assuming this scattering to originate from an unpaired electron with quantum numbers $S=1 / 2, L=2$, $J=5 / 2$, and $g(L J S)=1.2$, we find $n=12 \%$ at $T=326 \mathrm{~K}$ and $n=10 \%$ at $T=959 \mathrm{~K}$. If we assume the scattering to originate from an $s$ electron $[S=1 / 2, L=0, J=1 / 2$, and $g(L J S)=2]$, we find $n=48 \%$ and $n=42 \%$, respectively (see Table I). Thus, gallium displays a large magnetic cross section, but its magnitude appears to be only weakly temperature dependent.

More recently, the gallium dynamics has been investigated by means of inelastic neutron scattering [24], inelastic $\mathrm{x}$-ray scattering $[37,38]$, and molecular dynamics computer simulations [25], allowing us to verify the diffraction-based route and to obtain an independent estimate. The x-ray data, measuring the coherent cross section, demonstrated that the coherent contribution consists of three peaks (and three peaks only), whose linewidths and relative intensities change as a function of $q$ in a manner that can be modeled using the memory function formalism. Molecular dynamics computer simulations, which were in excellent agreement with the $\mathrm{x}$-ray data [37], showed that the self-dynamics (incoherent scattering) consists of a simple diffusion mode carrying practically all of the intensity (in neutron scattering) at low $q$ (99.6\% at $q=0.3 \AA^{-1}$ ) plus a cage-diffusion mode (intensity $0.4 \%$ at $q=0.3 \AA^{-1}$ ) whose importance increases [47] as $q^{2}$ for $q<1 \AA^{-1}$.

Interestingly, carefully corrected inelastic neutron experiments clearly showed the presence of a broad mode at 
$q=0.3 \AA^{-1}$, whose linewidth corresponded to the expected linewidth for cage duffusion, but whose intensity was observed to be as much as the intensity of the self-diffusion mode and easily exceeded that of the two propagating sound modes which carry about $80 \%$ of the intensity of the coherent contribution. Thus, there is a broad mode present in the neutron scattering data, which is not present in the x-ray scattering data and which exceeds the expected intensity based on simulations by at least two orders of magnitude. We identify this mode as originating from fluctuating magnetic moments on the $\mathrm{Ga}$ ions. We stress that, independent of whether our explanation for this additional intensity stands the test of further experiments or not, the fact remains that there is an additional mode present in liquid gallium, which at small momentum transfers scatters as many neutrons as the known incoherent and coherent scattering mechanisms. This mode is not reproduced in very accurate MD computer simulations. Using the numbers published in Ref. [24] in combination with Fig. 5 in this reference, we can use the strength of the self-diffusion peak to estimate the intensity of the broad quasielastic mode. Normalizing to the gallium incoherent cross $\operatorname{section}\left(\sigma_{i n c}=0.16 \mathrm{~b}\right.$ [51]), we find $\sigma_{\text {magn }}=0.4 \pm 0.1 \mathrm{~b}$ at $320 \mathrm{~K}$ and $\sigma_{\text {magn }}=0.5 \pm 0.1 \mathrm{~b}$ at $970 \mathrm{~K}$, below the estimates derived from the diffraction data, but similar in magnitude. These differences likely reflect the accuracy with which the magnetic contribution can be determined based on the diffraction data. The corresponding fraction of magnetic ions are given in Table I. Similar to the case for the diffraction data based estimates, we only observe a weak temperature dependence.

\section{DISCUSSION}

The available neutron scattering data reveal the presence of a broad quasielastic mode that is entirely absent in x-ray scattering and which cannot be ascribed to the standard mechanisms for incoherent and coherent scattering. We have argued that this mode is evidence for the existence of shortlived magnetic moments in nonmagnetic liquid metals. These moments come in and out of existence on the same time scale as the cage-diffusion motion, as observed in the quasielastic neutron scattering experiments on liquid $\mathrm{Hg}$ [1-3]. The alkali metals show only a weak effect, but the effect is much more pronounced in mercury and in group- 3 and -4 metals (see Table I).

The actual percentage of ions with unpaired electrons is more difficult to assess than establishing that such ions with unpaired electrons exist. For instance, it is feasible that the unpaired electron in liquid mercury is either an $s$ electron or a $d$ electron. The $6 s$ shell in mercury has been drawn in closer to the nucleus because of the relativistic contraction of the underlying shells, so it is definitely conceivable in a liquid that the $6 s$ shell can be completely filled (for some of the time at least). In other words, the observed paramagnetic intensity could originate from a $\mathrm{Hg}^{+}$or from a $\mathrm{Hg}^{3+}$ ion. (In liquid lead, it is in fact more likely that the paramagnetic contribution stems from $\mathrm{Pb}^{3+}$ than from $\mathrm{Pb}^{5+}$ ions, given the prevalence of lead to form $\mathrm{Pb}^{2+}$ in solids.) Should this indeed be the case, then the electrical resistance in liquid mercury does not come solely from electrons being scattered by ions, but also from electrons actually being captured by $\mathrm{Hg}$ ions; far from being unchanging, the Fermi sea constantly changes in size while interchanging electrons with the ions.

The phenomenon of the additional magnetic cross section seems to have been mostly overlooked. However, its implications on the interaction mechanisms in a liquid metal cannot be overlooked given the long range of the magnetic dipole interaction and the ability of localized moments to polarize the surrounding conduction electrons. In particular, it would be interesting to see how incorporation of paramagnetic ions and their polarization capability into the interatomic potential used in MD simulations would alter the characteristics of short-wavelength sound propagation.

Finally, this paramagnetic cross section provides a means of studying the cage-diffusion mechanism at small momentum transfers even in systems that do not exhibit an incoherent cross section, such as lead and aluminum. We are currently carrying out polarized neutron scattering experiments on liquid gallium in order to verify that the observed additional cross section is indeed magnetic in origin and to study its temperature dependence close to the solidification transition.

\section{ACKNOWLEDGMENTS}

We thank Dr. N. Iqbal for providing us with the diffraction data on aluminum, and we thank Dr. T. Scopigno and Dr. L.E. Bove for numerous discussions relating to their gallium data. Acknowledgment is made to the donors of the American Chemical Society Petroleum Research Fund for support of this research (Grant No. ACS PRF 42615-G10). K.S. is supported by a grant from the U.S. Department of Energy Office of Nuclear Energy, Science and Technology (No. DEFG07-03ID14531) to the Midwest Nuclear Science and Engineering Consortium under the Innovations in Nuclear Infrastructure and Education program.
[1] L. E. Bove, F. Sacchetti, C. Petrillo, B. Dorner, F. Formisano, and F. Barocchi, Phys. Rev. Lett. 87, 215504 (2001).

[2] Y. S. Badyal, U. Bafile, K. Miyazaki, I. M. de Schepper, and W. Montfrooij, Phys. Rev. E 68, 061208 (2003).

[3] L. E. Bove, F. Sacchetti, C. Petrillo, B. Dorner, F. Formisano, M. Sampoli, and F. Barocchi, Philos. Mag. B 82, 365 (2002);
J. Non-Cryst. Solids 307-310, 842 (2002).

[4] T. Bodensteiner, Chr. Morkel, W. Gläser, and B. Dorner, Phys. Rev. A 45, 5709 (1992).

[5] L. E. Bove, F. Sacchetti, C. Petrillo, and B. Dorner, Phys. Rev. Lett. 85, 5352 (2000).

[6] C. Cabrillo, F. J. Bermejo, M. Alvarez, P. Verkerk, A. Maira- 
Vidal, S. M. Bennington, and D. Martìn, Phys. Rev. Lett. 89, 075508 (2002).

[7] A. G. Novokov, M. N. Ivanovskii, V. V. Savostin, A. L. Shimkevich, O. V. Sobolev, and M. V. Zaezjev, J. Phys.: Condens. Matter 8, 3525 (1996).

[8] L. E. Bove, B. Dorner, C. Petrillo, F. Sacchetti, and J.-B. Suck, Phys. Rev. B 68, 024208 (2003).

[9] J. R. D. Copley and J. M. Rowe, Phys. Rev. A 9, 1656 (1974).

[10] D. I. Page, P. A. Egelstaff, J. E. Enderby, and B. R. Wingfield, Phys. Lett. 29A, 296 (1969).

[11] Chr. Morkel and W.-C. Pilgrim, J. Non-Cryst. Solids 312-314, 128 (2002).

[12] B. P. Alblas, W. van der Lugt, J. Dijkstra, W. Geertsma, and C. van Dijk, J. Phys. F: Met. Phys. 13, 2465 (1983).

[13] A. Stangl, C. Morkel, U. Balucani, and A. Torcini, J. NonCryst. Solids 205-207, 402 (1996).

[14] U. Balucani, A. Torcini, A. Stangl, and C. Morkel, Phys. Scr. T75, 13 (1995).

[15] A. Torcini, U. Balucani, P. H. K. de Jong, and P. Verkerk, Phys. Rev. E 51, 3126 (1995).

[16] H. Sinn and E. Burkel, J. Phys.: Condens. Matter 8, 9369 (1996).

[17] P. H. K. de Jong, P. Verkerk, and L. A. de Graaf, J. Phys.: Condens. Matter 6, 8391 (1994).

[18] S. J. Cocking, and P. A. Egelstaff, J. Phys. C 1, 507 (1968).

[19] D. M. North, J. E. Enderby, and P. A. Egelstaff, J. Phys. C 1, 784 (1968).

[20] O. Söderström, J. R. D. Copley, J.-B. Suck, and B. Dorner, J. Phys. F: Met. Phys. 10, L151 (1980).

[21] H. T. J. Reijers, W. van der Lugt, C. van Dijk, and M.-L. Saboungi, J. Phys.: Condens. Matter 1, 5229 (1989).

[22] N. Iqbal, N. H. van Dijk, V. W. J. Verhoeven, W. Montfrooij, T. Hansen, L. Katgerman, and G. J. Kearley, Adv. Mater. (Weinheim, Ger.) 51, 4497-4504 (2003).

[23] M. C. Bellissent-Funel, P. Chieux, D. Levesque, and J. J. Weis, Phys. Rev. A 39, 6310 (1989).

[24] L. E. Bove, F. Formisano, F. Sacchetti, C. Petrillo, A. Ivanov, B. Dorner, and F. Barocchi, Phys. Rev. B 71, 014207 (2005).

[25] L. E. Bove, F. Sacchetti, C. Petrillo, F. Formisano, M. Sampoli, and F. Barocchi, Philos. Mag. 84, 1609 (2004).

[26] H. Fredrikze, Phys. Rev. A 36, 2272 (1987).

[27] P. A. Egelstaff, N. H. March, and N. C. McGill, Can. J. Phys. 52, 1651 (1974).

[28] K. Tamura, M. Inui, I. Nakaso, Y. Oh'ishi, K. Funakoshi, and W. Utsumi, J. Phys.: Condens. Matter 10, 11405 (1998).

[29] K. Tamura and S. Hosokawa, J. Phys.: Condens. Matter 6,
A241 (1994).

[30] M. J. Huijben and W. van der Lugt, J. Phys. F: Met. Phys. 6, L225 (1976).

[31] W.-C. Pilgrim, S. Hosokawa, H. Saggau, H. Sinn, and E. Burkel, J. Non-Cryst. Solids 250-252, 96 (1999).

[32] T. Scopigno, U. Balucani, G. Ruocco, and F. Sette, Phys. Rev. E 65, 031205 (2002).

[33] T. Scopigno, U. Balucani, G. Ruocco, and F. Sette, Phys. Rev. Lett. 85, 4076 (2000).

[34] M. Dzugutov, K.-E. Larsson, and I. Ebbsjo, Phys. Rev. A 38, 3609 (1988).

[35] IAMP database of SCM-LIQ, Tohoku University. Available at http://www.tagen.tohoku.ac.jp/general/building/iamp/database/ $\mathrm{scm} / \mathrm{LIQ} / \mathrm{sq} \cdot \mathrm{html}$

[36] T. Scopigno, U. Balucani, G. Ruocco, and F. Sette, Phys. Rev. E 63, 011210 (2000).

[37] T. Scopigno, A. Filipponi, M. Krisch, G. Monaco, G. Ruocco, and F. Sette, Phys. Rev. Lett. 89, 255506 (2002).

[38] T. Scopigno, R. Di Leonardo, L. Comez, A. Q. R. Baron, D. Fioretto, and G. Ruocco, Phys. Rev. Lett. 94, 155301 (2005).

[39] U. Balucani and M. Zoppi, Dynamics of the Liquid State (Clarendon Press, Oxford, 1994), and references therein.

[40] I. M. de Schepper, E. G. D. Cohen, and M. J. Zuilhof, Phys. Lett. 101A, 399 (1984).

[41] E. G. D. Cohen, P. Westerhuijs, and I. M. de Schepper, Phys. Rev. Lett. 59, 2872 (1987).

[42] P. N. Pusey, H. N. W. Lekkerkerker, E. G. D. Cohen, and I. M. de Schepper, Physica A 164, 12 (1990).

[43] R. Verberg, I. M. de Schepper, and E. G. D. Cohen, Europhys. Lett. 48, 397 (1999); Phys. Rev. E 61, 2967 (2000).

[44] G. L. Squires, Introduction to the Theory of Thermal Neutron Scattering (Dover, New York, 1996).

[45] V. F. Sears, Adv. Phys. 24, 1 (1975).

[46] Handbook of Chemistry and Physics, 79th ed. (CRC, Boca Raton, 1999).

[47] The relative intensity $I_{r e l}$ of the cage-diffusion mode, as observed in MD simulations, is given at low $q$ values by $(q \Delta)^{2} / 10$ with $\Delta$ the radius of the cage. This is true for both the coherent and incoherent contribution since it is a formfactor effect: $I_{\text {rel }}=\int_{\text {cage }} e^{i \vec{q} \cdot \vec{r}} d \vec{r} / V_{\text {cage }}$.

[48] G. S. Dubey, R. Bansal, and K. N. Pathak, J. Phys. C 13, 6119 (1980).

[49] T. Gaskell, J. Phys. F: Met. Phys. 16, 381 (1986).

[50] N. M. Keita and S. Steinemann, J. Phys. C 11, 4635 (1978).

[51] V. F. Sears, Neutron News 3(3), 29 (1992); also http:// www.ncnr.nist.gov/resources/n-lengths/. 